Optical Society of America. Daniel Post, "Moiré interferometry with white light," Appl. Opt. 18, 4163-4167 (1979). doi: 10.1364/ao.18.004163

\title{
Moiré interferometry with white light
}

\author{
Daniel Post
}

\begin{abstract}
While high-sensitivity moiré interferometry requires monochromatic light, an auxiliary compensator grating removes the requirement. Experimental verification utilized white light for moiré interference of sensitivity $0.833 \mu \mathrm{m} /$ fringe. Configurations readily amenable to compensation include moiré fringe multiplication by even factors and the minimum-deviation arrangement. Chromatic compensation is effective with virtual reference gratings as well as real reference gratings; in both cases the moire interference system becomes achromatic. Compensation provides the special quality of nearly zero phase difference-an actual range of $\pm \pi$-between interfering beams. Potential applications include use of electrical spark light sources for high-sensitivity dynamic moiré photography.
\end{abstract}

\section{Introduction}

An important method of moire interferometry is illustrated in Fig. 1. Two gratings, called reference and active gratings, of frequency $f$ and $f / 2$ (rulings per millimeter), respectively, are illuminated obliquely by a collimated beam. Component beams of diffractionorder sequence 0,1 and $1,-1$ are collected and observed. The resultant two-beam interference pattern depicts in-plane displacements of the coarser active grating with a sensitivity of $1 / f \mathrm{~mm} /$ fringe; the moire fringe multiplication factor is $2 .{ }^{1}$

Monochromatic light is used. Otherwise, rays of central wavelength $\lambda$ emerge normal to the gratings, but rays of wavelength $\lambda \pm \Delta \lambda$ emerge in different directions, $\pm \phi$, respectively. If the spectral bandwidth is not very small, two main problems arise: the aperture of the observing system must be large to accommodate light emerging in the $\pm \phi$ directions; and the desired symmetry of rays in the gap between the gratings, which is responsible for insensitivity to out-of-plane displacements, is violated.

Curiously, both factors respond to a chromatic compensation technique that enables use of any spectral bandwidth, including white light.

The author is with Virginia Polytechnic Institute \& State University, Engineering Science \& Mechanics Department, Blacksburg, Virginia 24061 .

Received 20 August 1979.

0003-6935/79/244163-05\$00.50/0.

(C) 1979 Optical Society of America.

\section{Compensation}

The compensation scheme is illustrated in Fig. 2. Collimated chromatic light is first passed through an auxiliary or compensator grating of frequency $f / 2$. With normal incidence on the compensator, the angle of emergence of the -1 diffraction order is

$$
\alpha=-\sin ^{-1} \frac{\lambda f}{2}
$$

This is the angle of incidence on the reference grating of frequency $f$, and for this grating it is precisely the right angle to generate symmetrical zeroth and firstorder diffraction angles, namely, $-\alpha$ and $+\alpha$, respectively, for any wavelength $\lambda$.

Proceeding through the active grating of frequency $f / 2$, rays of diffraction-order sequence 0,1 are bent by angle $\alpha$, while those of sequence $1,-1$ are bent by $-\alpha$. The emergent rays become parallel and also parallel with the beam that is incident upon the compensator.

Equation (1) applies for every wavelength. Angle $\alpha$ is a function of wavelength $\lambda$, and it varies with $\lambda$ exactly as required for symmetry and common emergence direction. As distinct from Fig. 1, the systematic variations of angle of incidence on the grating pair overcome the aforementioned problems.

Sensitivity is a function of $f$ alone, and sensitivity, in displacement per fringe, is the same for all wavelengths. For a continuous source, the result is a continuum of identical interference patterns in each wavelength, all identically superimposed. The system is achromatic. 


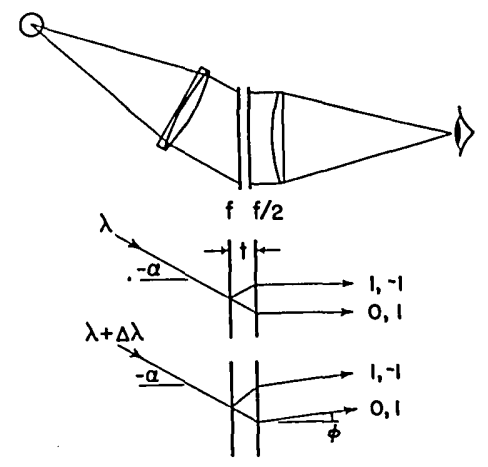

Fig. 1. Optical arrangement and pertinent paths for moiré fringe multiplication by a factor of 2 .

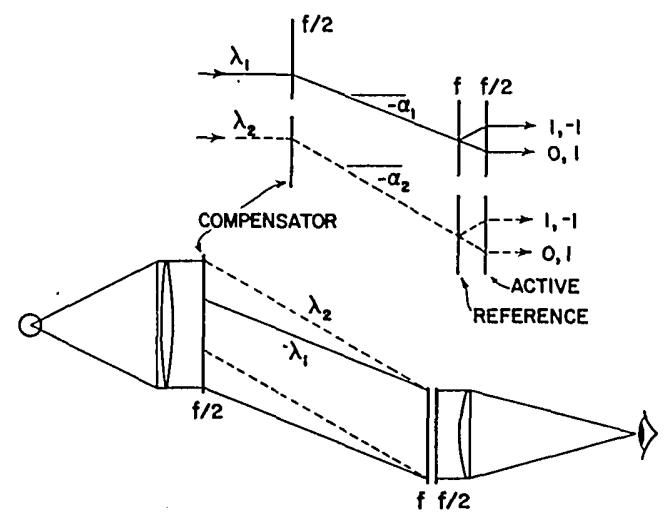

Fig. 2. Pertinent paths and optical arrangement with compensation for chromatic light.
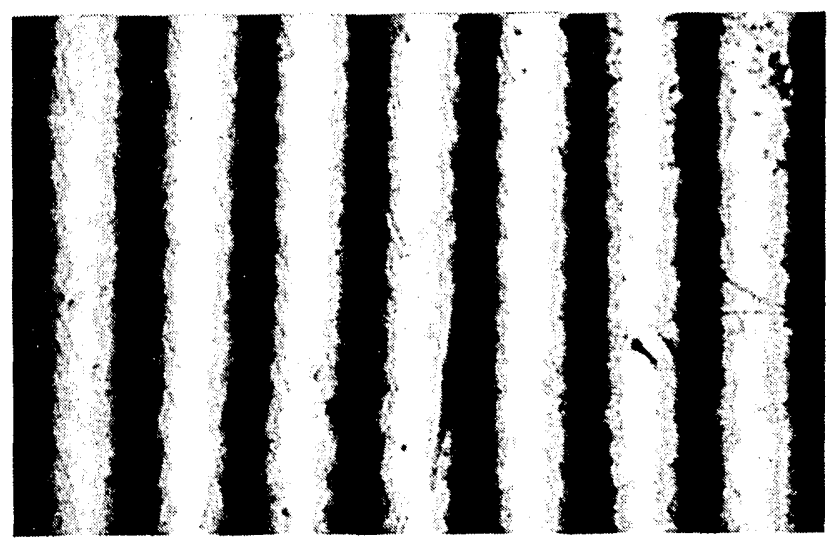

Fig. 3. White-light moiré interference pattern by method of Fig. 2; $f=1200$ rulings $/ \mathrm{mm}$.

\section{Experimental Verification}

Figure 3 is a white-light moiré interference pattern produced by the method of Fig. 2. Grating frequency $f$ was 1200 rulings/mm (30,480/in.); grating size was 5 -cm diam., although a smaller zone of the moiré grating pair was illuminated in all wavelengths of the -1 diffraction order from the compensator; the gap between reference and active gratings was $1 / 4 \mathrm{~mm}$; the gap between compensator and reference gratings was $\sim 7 \mathrm{~cm}$. The gratings were all of the blazed type, with the compensator exhibiting strong diffraction in the -1 order and very weak diffraction in the -2 order. The reference grating of frequency $f$ exhibited approximately equal strengths in the 0 and 1 orders for incidence at $-\alpha$, and it exhibited very weak diffraction in the -1 and 2 orders in the red and none in the blue. The active moiré grating exhibited approximately symmetrical diffraction strengths about the zeroth order. (Gratings used were Bausch \& Lomb catalog nos. 35-54-05-520, 3554-05-540, and 35-54-05-570, respectively.) Illumination was an unfiltered incandescent light source, and photography was on Polaroid type-57 film (panchromatic). The photograph covers an area of $\sim 27 \mathrm{~mm} \times$ $17 \mathrm{~mm}$ of the moire gratings.

The moire fringes are perpendicular to the rulings and show displacements of rigid body rotation. Sensitivity is $1 / f \mathrm{~mm} /$ fringe, or $0.833 \mu \mathrm{m} /$ fringe. In terms of wavelength, sensitivity is $\sim 1.5$ wavelengths of green light per fringe. Resolution of detail is good, with disturbances associated with small periodic errors of the gratings visible in the fringes.

Under ideal circumstances the fringes would appear black and white, but these actually appeared with a blue tint in the dark bands and a pink tint in the bright bands. This color is associated with grating characteristics and variations of blaze efficiencies with wavelength. Interference was most complete toward the red end of the spectrum, enhancing the red in the constructive interference zones and transmitting blue background in the destructive interference zones.

\section{Grating Characteristics}

The reference and active gratings should have the same characteristics as required for two-beam interference in the uncompensated method. It is worth noting that two-and only two-diffraction orders emerge from the reference grating if frequency is in the range

$$
\frac{2}{\lambda}>f \geqq \frac{2}{3 \lambda}
$$

or grating pitch $g$ is

$$
\frac{\lambda}{2}<g \leqq \frac{3 \lambda}{2} .
$$

For green light, this frequency range is $\sim 1200-3600$ rulings $/ \mathrm{mm}(30,000-90,000 /$ in. $)$. Strictly two-beam interference is assured for moire interferometry in this range.

The compensator grating must exhibit a significant intensity of diffraction order -1 over the spectral zone 
employed. When the full visible spectrum is employed, intensity of diffraction order -2 must be small compared with that of -1 to avoid overlapping orders in the location of the moiré gratings.

For any spectral range, a diagram can be made showing the beams of extreme wavelengths $\lambda_{1}$ and $\lambda_{2}$ emerging from the compensator for each diffraction order. The reference and active gratings should be located where the two extreme first-order beams coexist alone without overlapping of other orders. The optimum distance between compensator and reference gratings and the maximum useful width of moiré gratings can be determined from the diagram.

\section{Zero Phase Difference}

In Fig. 4, let $W$ represent the wave front of any monochromatic element of the incident collimated beam. After traveling through the system in diffraction sequences 0,1 and $1,-1$, components of $W$ appear at a later time as wave fronts $W_{0}$ and $W_{1}$, respectively. However, they traveled exactly equal path lengths in that time interval and therefore lie in a common plane. Their path difference and phase difference are zero.

This result is characteristic of the present compensation method. In the case of the uncompensated system of Fig. 1, with monochromatic light, the path difference $\delta$ between the 0,1 and $1,-1$ wave fronts may be derived by a geometrical analysis, with the result

$$
\delta=t f \lambda \tan \alpha .
$$

Here the path difference is of the same order as gap thickness $t$ when high-frequency gratings are used, and phase difference $2 \pi \delta / \lambda$ may be very large. Yet, with compensation, $\delta=0$.

The foregoing pertains to a null field-when moiré fringe order is everywhere zero. Let the active grating be deformed nonhomogeneously by the action of a strain field. Now its frequency varies from point to point, and its alignments with the direction of rulings of the reference grating also varies from point to point in the field. As a result, wave fronts $W_{0}$ and $W_{1}$ emerge as warped surfaces.

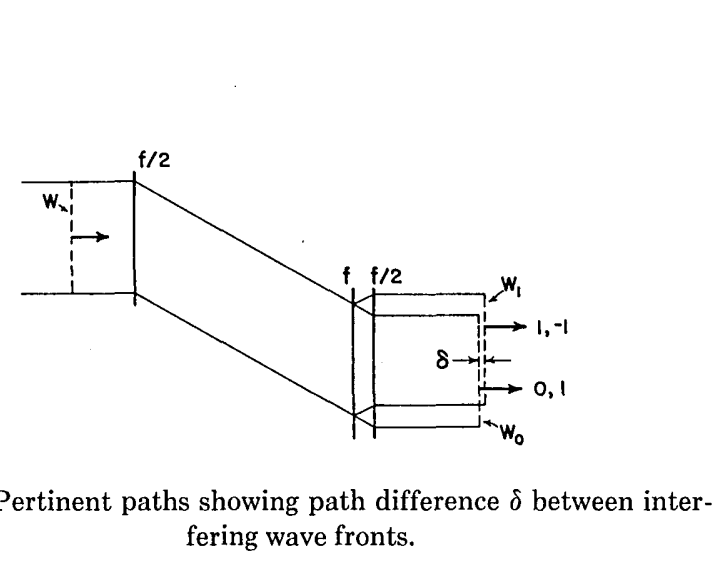

Fig. 4. Pertinent paths showing path difference $\delta$ between interfering wave fronts.
Assuming that the strain or deformation varies from point to point in a continuous fashion, the warped wave fronts $W_{0}$ and $W_{1}$ must be smoothly warped surfaces. The moiré fringe order (or interference fringe order) must vary as a continuous function across the field of view.

This moire technique is a member of a large family of two-beam interference methods. In general, an interferogram is a contour map of the absolute separation between the two emergent wave fronts. However, with moiré interferometry (and unlike other types of twobeam interferometry), the phrase "absolute separation between the two emergent wave fronts" has no meaning. At every point of constructive interference in the moiré pattern, the rulings in the reference and active gratings are in identical registration and yield identical interference effects. The phase difference $2 \pi \delta / \lambda$ between wave fronts is identical in all zones of constructive interference; in all zones of destructive interference, the phase difference is $2 \pi \delta / \lambda \pm \pi$. Consequently, the familiar proportionality between phase difference and fringe order does not apply to moiré interferometry. Instead, phase difference is everywhere within the range $2 \pi \delta / \lambda \pm \pi$, where $\delta$ is given by Eq. (4) for common moiré interferometry and $\delta=0$ when compensation is used.

Thus, the concept of contour lines depicting the relative separation between wave fronts is useful for describing the origin of moire interference fringes, while the usual relationship between fringe order and phase difference (or path difference) is invalid. The remarkable quality of moiré interferometry with compensation is that phase difference is within the range $\pm \pi$-nearly zero phase difference-regardless of moiré fringe order.

\section{Other Configurations}

\section{Multiplication by Even Factors}

The moiré interferometry method discussed above is a specific case of the moire fringe multiplication described in Ref. 1. The present compensation technique

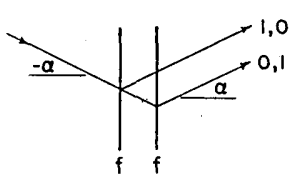

(a)

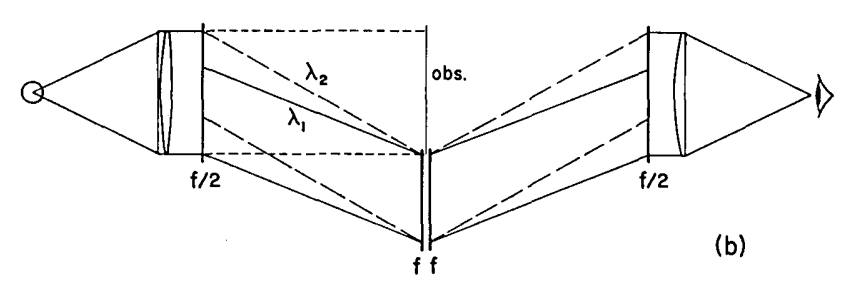

Fig. 5. Compensation with minimum deviation configuration; obs. is obstruction to stop beam of zero diffraction order. 
can also be used in precisely the way described above for other fringe multiplication factors $\beta$, where $\beta$ is an even integer. Grating frequencies would be $f / 2$ for the compensator, $f$ for the reference, and $f / \beta$ for the active grating.

\section{Minimum Deviation}

The moiré interferometry configuration of minimum deviation, discussed extensively by Guild, ${ }^{2}$ utilizes reference and analyzer gratings of equal frequency; the multiplication factor is unity. Symmetrical angles of incidence $(-\alpha)$ and emergence $(\alpha)$ are employed, as shown in Fig. 5(a), and inverted diffraction sequences 0,1 and 1,0 are collected and observed.

Chromatic compensation for this case is illustrated in Fig. 5(b). The left side of the diagram is identical to that in Fig. 2, providing symmetrical paths in the gap between moiré gratings for every wavelength. A second compensator grating is employed to the right of the moiré gratings to bend the emergent beams into a common direction, regardless of wavelength.

\section{Virtual Gratings}

A steady-state system of walls of constructive and destructive interference is formed where two coherent collimated beams cross in space. The walls are uniformly spaced, with frequency $f$ given by Eq. (1), where $\alpha$ is the half-angle between the intersecting beams. This interference system is denoted by the author as a virtual grating, since it has exactly the same composition as the light of zeroth and first diffraction orders exiting from the real reference grating in Fig. 1.

Numerous optical systems can be devised to produce a virtual grating. A simple one is illustrated in Fig. 6 . The top half of a monochromatic collimated beam enters the hatched zone at angle $-\alpha$, while the bottom half enters, after reflection by the mirror, at angle $+\alpha$. The virtual grating is the interference pattern in the hatched zone. While possessing the virtue of simplicity, this technique produces a virtual grating of maximum height smaller than the collimator diameter.

If light with a continuous spectrum between $\lambda_{1}$ and $\lambda_{2}$ is used in Fig. 6, a continuum of frequencies $f$ occurs, according to Eq. (1). Moiré requires a reference grating of fixed frequency, and unless $\lambda_{2}-\lambda_{1}$ is very small, this system would not produce moiré interference.

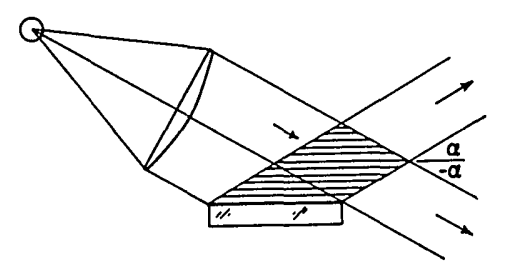

Fig. 6. Virtual grating produced by two monochromatic beams crossing at angles $\pm \alpha$.
However, compensation can be used with a broad spectrum to produce a virtual grating of fixed frequency $f$ and to produce moire interference. The system is depicted in Fig. 7. A collimated chromatic beam illuminates the compensator grating of frequency $f / 2$, and light from its diffraction order -1 produces the virtual grating in the hatched zone. Again, the compensator produces exactly the right angle $\alpha$ in each wavelength to produce a virtual grating of frequency $f$; the virtual grating is achromatic.

When an active grating of frequency $f / 2$ intercepts the virtual grating (Fig. 7), the system is analogous to that of Fig. 2. Light diffracted in the +1 and -1 orders of the active grating emerges normal to the grating to produce moiré multiplication by a factor of 2 .

Virtual gratings can be produced by many other optical schemes, and many of these can be modified by the compensation technique to enable use of chromatic light. Also, compensated virtual gratings can be used as the reference grating for moire multiplication by other even factors $\beta$ or for moiré by the minimumdeviation technique.

\section{Discussion}

In all the configurations discussed here, the compensation techniques cause the incident and emergent beams to assume parallel directions of propagation. This condition results in zero path difference between interfering beams for the null field and phase differences in the minute range $\pm \pi$ for the general moire interferogram.

Without compensation, the path difference between interfering beams is given by Eq. (4) for moiré multiplication by even factors and also for minimum deviation using real reference gratings. For moiré multiplication by even factors using a virtual reference grating, the path difference between interfering wave fronts is proportional to distance $y$ (Fig. 7), the distance across the virtual grating from its point of zero interference fringe order, namely,

$$
\delta=\lambda f y,
$$

and $\delta$ may be very large for practical situations. Yet, with compensation, the special quality of zero path difference is achieved.

The moiré systems illustrated here are all transmis-

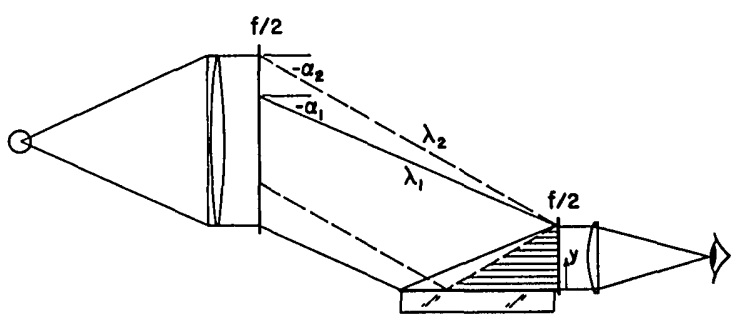

Fig. 7. Optical arrangement for moiré interferometry with chromatic light and virtual reference grating. 
sion schemes, requiring the active gratings to be transmission type on transparent substrates. Frequently, however, the substrate must be opaque, and a reflection system is required. Reflection counterparts exist for all the transmission methods discussed above. Illumination on the active grating is the same in each case', but the active grating is a reflection grating. In Figs. 2 and 7, the light emerging normal to the grating on the reflection side is collected and observed. In Fig. 5, light returning along the path of incidence is observed, and this is done by installing a beam splitter between the source and collimating lens to view the moiré pattern.

While compensation for chromatic light has been accomplished, the requirements on source width, or degree of collimation, are unaltered. Sources of extremely narrow dimensions must be used when the gap between reference and active gratings is large or when large virtual gratings are used.
Compensation is a fascinating academic concept, but numerous practical applications also appear imminent. The author's immediate interest stems from his desire to use electrical discharge sparks as multiple light sources for dynamic moiré photography. The broad spectrum of a spark in air would violate the requirements of high-sensitivity moire interferometry, but compensation provides the desired compatibility.

This work was supported by the National Science Foundation under Grant ENG-7824609 with Clifford J. Astill as NSF program director. This support, as well as aid from staff and facilities of the Engineering Science and Mechanics Department of VPI\&SU, is greatly appreciated.

\section{References}

1. D. Post, Appl. Opt. 10,901 (1971).

2. J. Guild, The Interference System of Crossed Diffraction Gratings (Clarendon Press, Oxford, 1956).

The 35 th Annual Symposium on Molecular Spectroscopy will be held at the Ohio State University in Columbus, Ohio, between June 16-20, 1980. The deadline for the receipt of contributed papers is March 1, 1980. If you wish to present papers you should submit the titles and abstracts in the required format which can be obtained from Professor K. Narahari Rao, MOLECULAR SPECTROSCOPY SYMPOSIUM, Department of Physics, The Ohio State University, 174 West 18 th Avenue, Columbus, Ohio, 43210, U.S.A. If you are on the Symposium Mailing List you will automatically receive the instructions for preparing the titles and abstracts by the end of 1979. Please write to Professor Rao for details, only if you have not been receiving Symposium announcements in the past 